\title{
Growth mechanisms for InAs/GaAs QDs with and without Bi
}

\section{surfactants}

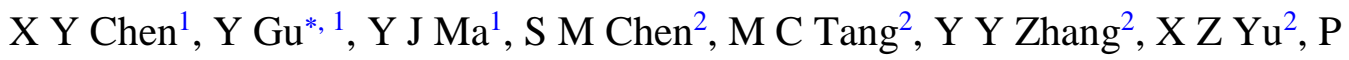

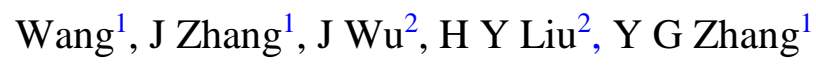 \\ ${ }^{1}$ State Key Laboratory of Functional Materials for Informatics, Shanghai Institute of Microsystem \\ and Information Technology, Chinese Academy of Sciences, Shanghai 200050, China \\ ${ }^{2}$ Department of Electronic and Electrical Engineering, University College London, London WC1E \\ 7JE, $U K$
}

Abstract: We report systematic study of growth of self-assembled InAs quantum dots (QDs) on GaAs substrate at various temperatures with and without exposure of bismuth surfactants. Results show that the coalescence amongst InAs QDs is considerably inhibited by the exposure of bismuth flux during growth in the temperature range from 475 to $500{ }^{\circ} \mathrm{C}$, leading to improved dot uniformity and a modified dot density. The mechanism of the suppression effect by bismuth surfactants on the strain-induced islanding through inhibiting the indium adatom mobility and the evaporation rate on the surface kinetically is thus clarified for the growth of InAs QDs. The photoluminescence peak wavelength for the InAs QDs with Bi exposure red shifted slightly due to the suppression of $\mathrm{Bi}$ atoms on the QD dissolution during the capping process at higher temperatures. Moreover, by investigating the temperature-dependent quenching processes with and without $\mathrm{Bi}$ exposure, it is observed that the the weak carrier confinement occurred in QDs with the presence of Bi caused broadness in the linewidths.

\footnotetext{
${ }^{*}$ Corresponding author. Tel.: +86-21-62511070; fax: +86-21-52419931. 
Keywords: InAs quantum dots; Bismuth; Surfactant; Molecular beam epitaxy 


\section{Introduction}

Owing to the small segregation coefficient and low solid solubility in host lattice [1], bismuth (Bi) is considered as an ideal surfactant for the material growth and has recently been widely studied in different systems such as III-V quantum dots (QDs)

[2], quantum wells (QWs) [3], heteroepitaxy of Ge on Si [4], and Co/Cu multilayers [5], etc. Most of these researches are devoted to improve the perfection of structures in aspects of ordering, interfaces, and surfaces. Self-assembled InAs QDs on GaAs (001) are favorable for devices operating in the optical telecom wavelength bands as the improved height/diameter aspect provides a deeper confinement of the charge carriers as compared to other nanostructures [6-8]. Given that QD-based device performances will be affected by the dot density and dimension, the QD size, uniformity, and density should be well tuned to guarantee optimum performances of QD-based devices. For example, an improvement in QD density and uniformity has the potential to increase the differential gain and modulation bandwidth for QD lasers as well as the interband and intersubband absorption strength for QD-based photodetectors. While the preserve of the shape and the improvement of uniformity of InAs QDs using Bi surfactants have been verified in previous works either by molecular beam epitaxy (MBE) or metal organic chemical vapor deposition (MOCVD) $[9,10]$, whether $\mathrm{Bi}$ decreases or increases the surface migration of indium (In) adatoms during the growth is still unclear since the effect of $\mathrm{Bi}$ surfactants during InAs QD growth has been interpreted inconsistently. Some researchers hold the point that $\mathrm{Bi}$, acts as a reactive surfactant, can kinetically limit the surface adatom mobility 
and decrease the In adatom diffusion length and thus increase the QD density $[9,10]$. Others argue an opposite effect of Bi on the QD density and dimension [11]. Since the QD density of uncapped QDs is greatly affected by not only the In adatom diffusion length but also by the evaporation rate of InAs nuclei on the surface. Both processes can be influenced by presence of Bi atoms and depends strongly on temperature. That would be the reason why the controversy exists.

Here, we reconcile these apparent contradictions by comparing InAs QDs grown with and without $\mathrm{Bi}$ exposure over the temperature range from 475 to $500{ }^{\circ} \mathrm{C}$. It is observed that the Bi surfactant leads to a decrease in QD density for lower growth temperatures $\left(475-485{ }^{\circ} \mathrm{C}\right)$, but an increase for higher growth temperatures (492 $\left.500{ }^{\circ} \mathrm{C}\right)$. The variations of the dot density are ascribed to the suppressing effect of $\mathrm{Bi}$ surfactants on the In adatom surface diffusion length during MBE growth regardless of growth temperatures, as is expected for a reactive surfactant [12]. Moreover, using Bi as a surfactant enables an improvement in QD uniformity, suggesting that the Bi surfactant-mediated growth of QDs has the potential to improve the performance of QD-based optoelectronic devices.

\section{Experiments}

All the InAs/GaAs QD samples were grown by gas-source $\mathrm{MBE}$ in a VG Semicon V90H system on semi-insulating GaAs (001) substrates. The structures were started with a $200 \mathrm{~nm}$ GaAs buffer layer grown at $580{ }^{\circ} \mathrm{C}$ after desorption of the native oxide layer. Then the substrate temperature was ramped down for the growth of the first layer of InAs QDs with a thickness of nominal 2.8 monolayers (MLs) and 
deposition rate of $0.07 \mathrm{ML} / \mathrm{s}$. This QD layer was used for photoluminescence (PL) measurements. A $10 \mathrm{~nm}$ GaAs capping layer was grown immediately at the same temperature. Then the temperature was ramped up to $580{ }^{\circ} \mathrm{C}$ for the deposition of a $110 \mathrm{~nm}$ GaAs spacing layer. At last, the InAs QD layer was repeated on the surface of the GaAs spacer for dot morphology studies. To investigate the effect of Bi exposure on the InAs QDs, the Bi shutter was only opened during the deposition of QDs with the Bi flux of $1.09 \mathrm{nA}$ (Beam equivalent pressure $\sim 8.8 \times 10^{-9}$ torr), which has been proved to be helpful for the growth of triangular highly strained InAs/InGaAs QWs on InP [3], otherwise the Bi shutter remained closed during the growth. The substrate temperatures of $475,485,492$ and $500{ }^{\circ} \mathrm{C}$ were adopted for the deposition of InAs QD layers. At such relatively high growth temperatures, Bi atoms tended to segregate, floated on the growth surface without incorporating into the host material [13], and acted evidently as a reactive surfactant. For comparison, another set of InAs QD samples without $\mathrm{Bi}$ exposure was also grown on GaAs under nominally identical growth conditions. Note that no deliberate growth interruption was used for both sets of samples after the deposition of InAs QDs in an attempt to maintain the similarity between the buried QDs and the uncapped QDs to the utmost, as well as to avoid artificial impacts on the surface adatom (especially In) diffusion length. In this way, the contrast between the two sets of QDs with and without Bi exposure would also be enhanced. The nominal growth rate of GaAs was $0.16 \mathrm{~nm} / \mathrm{s}$. The V/III ratio was far higher than 20 in this experiment according to our previous calibration, and $\mathrm{As}_{2}$ species were used for all layers. 


\section{8}

microscope $(\mathrm{AFM})$ in the contact mode. The scanned area was $1 \times 1 \mu \mathrm{m}^{2}$. PL spectra were measured with a Nicolet IS50 Fourier transform infrared spectrometer (FTIR).

Samples were excited by a diode-pumped solid state (DPSS) laser $(\lambda=532 \mathrm{~nm})$.

Temperature-dependent PL measurements were carried out by mounting samples into a continuous-flow helium cryostat, and a Lake Shore 330 temperature controller was used to adjust the temperature from 10 to $300 \mathrm{~K}$. The laser spot area was about $4.5 \times$ $10^{-2} \mathrm{~cm}^{2}$. The laser power used in RT and temperature-dependent PL measurements were about 340 and $136 \mathrm{~mW}$, respectively.

\section{Results and Discussion}

(1)
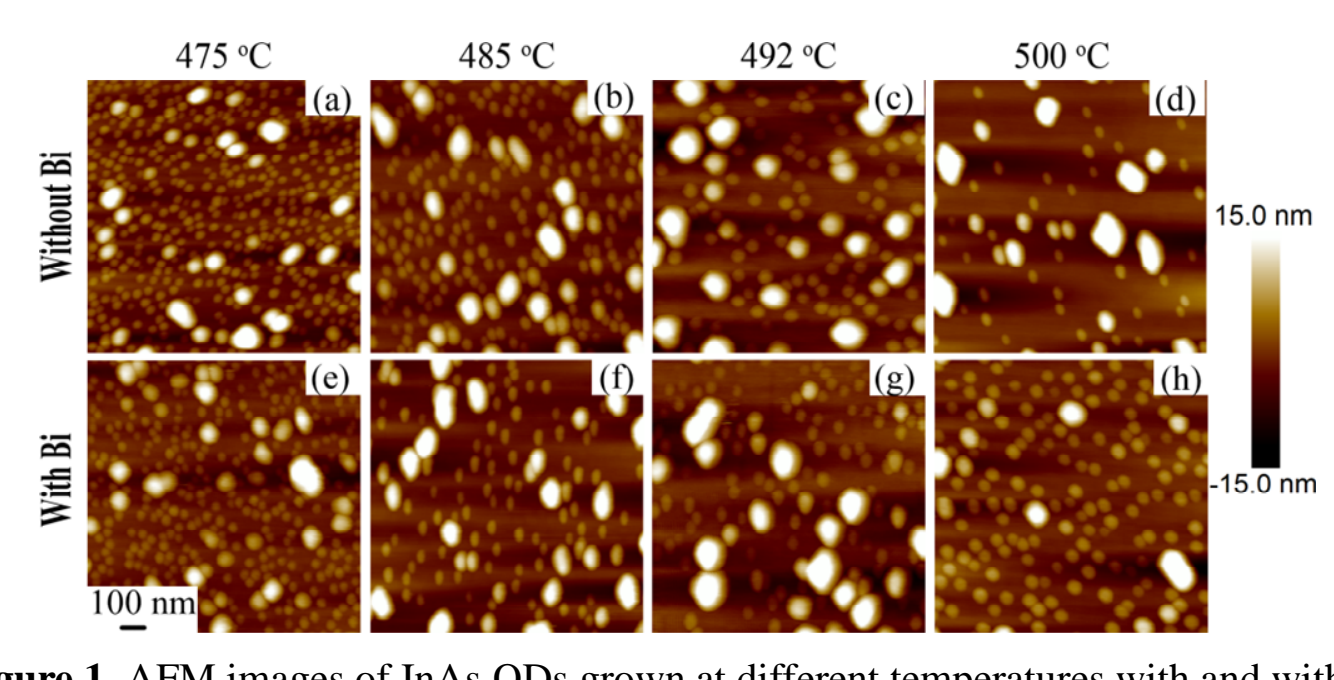

Figure 1. AFM images of InAs QDs grown at different temperatures with and without Bi exposure.

Topographical AFM images of the grown InAs QDs without and with Bi exposure are shown in figure 1 . For the case of Bi-unmediated samples, at low substrate temperatures, the surface population of free In adatoms diffused relatively 
slowly due to a low surface energy and the average diffusion distance was short before they combined with dissociated As2 molecules. This results in a high dot density with a small lateral size in general. At an elevated temperature, their diffusion distances elongated, and dots coalesced with each other intensely with an increase in surface energy of In adatoms, thus the dot density declined. As a concomitance of the dot growing up, the random aggregation of islands increased, corresponding to the piling up of bright circular features in figures 1(b) and 1(c). At the highest substrate temperature of $500{ }^{\circ} \mathrm{C}$, it was observed that big dots evolved into larger islands leaving a few scattered dots surrounded, mostly caused by desorption of uncapped QDs and Ostwald ripening, as shown in figure 1(d). However, dissimilarities occurred in both the dot density and dimension for the case of Bi-mediated samples, as shown in figures 1(e)-1(h). The main difference can be found in the variation of dot densities in comparison to that without the $\mathrm{Bi}$ exposure ranging from 475 to $500{ }^{\circ} \mathrm{C}$. The InAs dots formed under Bi exposure not only showed reduced variations in the dot densities but also became more homogeneous especially in the case of $500{ }^{\circ} \mathrm{C}$, where the dislocated islands significantly suppressed, opposite to those without Bi exposure. These results indicate that the $\mathrm{Bi}$ surfactants significantly modified the growth kinetics of QDs. Bi atoms can preserve the dot dimension and density during cooling down, similarly to the property of $\mathrm{Sb}$ atoms $[14,15]$. 


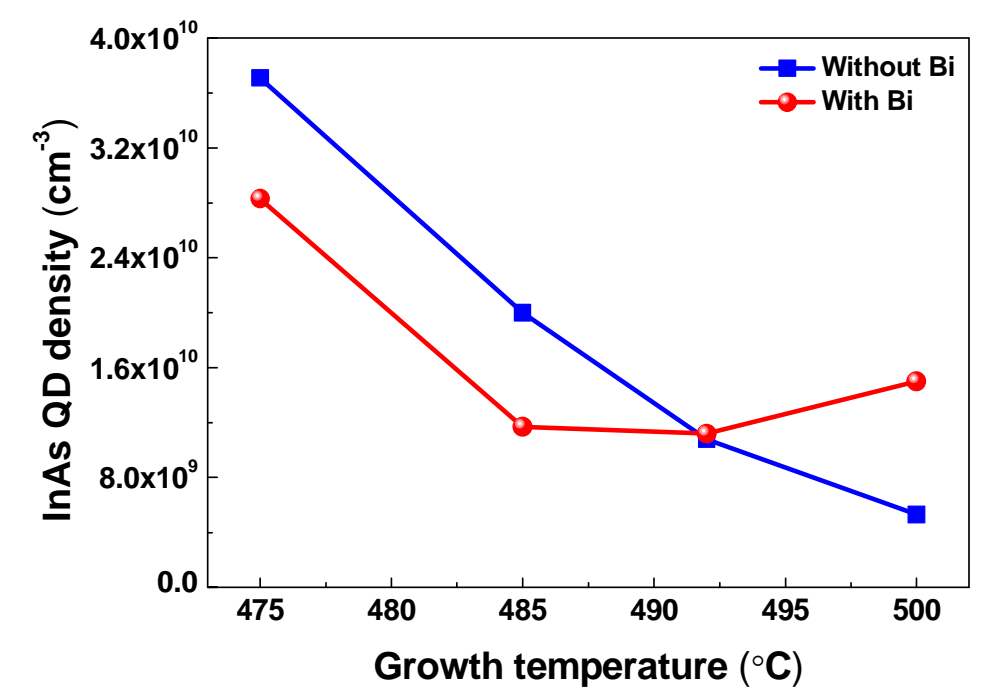

107

108

109

110

111

112

113

114

To quantify the influence of the $\mathrm{Bi}$ exposure on the QD densities, temperature-dependent InAs QD densities with and without Bi exposure were plotted in figure 2. Without Bi, the uncapped InAs QD densities decreased monotonically with the deposition temperature increasing from 475 to $500{ }^{\circ} \mathrm{C}$ mostly caused by the increase in desorption rates of In adatoms since they have additional time to change en for surface changes during cooling down, especially for samples grown at higher temperatures. Nevertheless, the dot dimension and density of buried QDs can be quite different. It may be much higher in both size and dot density but smaller in size! However, when Bi exposure was used, the variation of the QD density differed considerably over the whole temperature range of $475-500{ }^{\circ} \mathrm{C}$. It is obvious that the Bi-mediated QD ripening process during cooling down is suppressed by Bi atoms. As a result, QDs with Bi exposure showed a decrease in the overall density at low 
temperatures but an increase at high temperatures compared to the QDs without $\mathrm{Bi}$ exposure.

It is well known that the InAs dot density and dimension depend strongly upon factors such as the surface diffusion length of In adatoms [16], the desorption rate of In adatoms from the substrate surface and the dissociation rate for the tiny InAs nuclei [17]. It is supposed that the dominant factors for QD density and dimension should be varied over the variation range of temperatures, which will be strengthened by the employment of $\mathrm{Bi}$ surfactants for the QD growth. At low temperatures, little desorption of In adatoms and dissociation for the tiny InAs nuclei occur. The dot density is probably dominated by the diffusion length of In adatoms on surface, and the reduction of the surface diffusion length of In adatoms due to the presence of $\mathrm{Bi}$ undoubtedly will defer the InAs QD formation by delaying the onset of QDs, leading to a decrease in the overall QD density with respect to the case without Bi exposure. At high temperatures, the desorption of In adatoms from the growth surface and the dissociation for the tiny InAs nuclei aggravate intensely, but they are ultimately suppressed by the presence of Bi. While the suppression effect of Bi exposure on the dot coalescence still exists due to the restrained surface diffusion length of In adatoms, thus leading to a higher QD density instead compared to that without Bi exposure.

These results demonstrate the suppression effect of $\mathrm{Bi}$ surfactants on the In adatom surface diffusion, which eventually leads to a suppression of QD coalescence and ripening, similar to the behavior of antimony ( $\mathrm{Sb}$ ) surfactants for QD growth [18, 19]. 

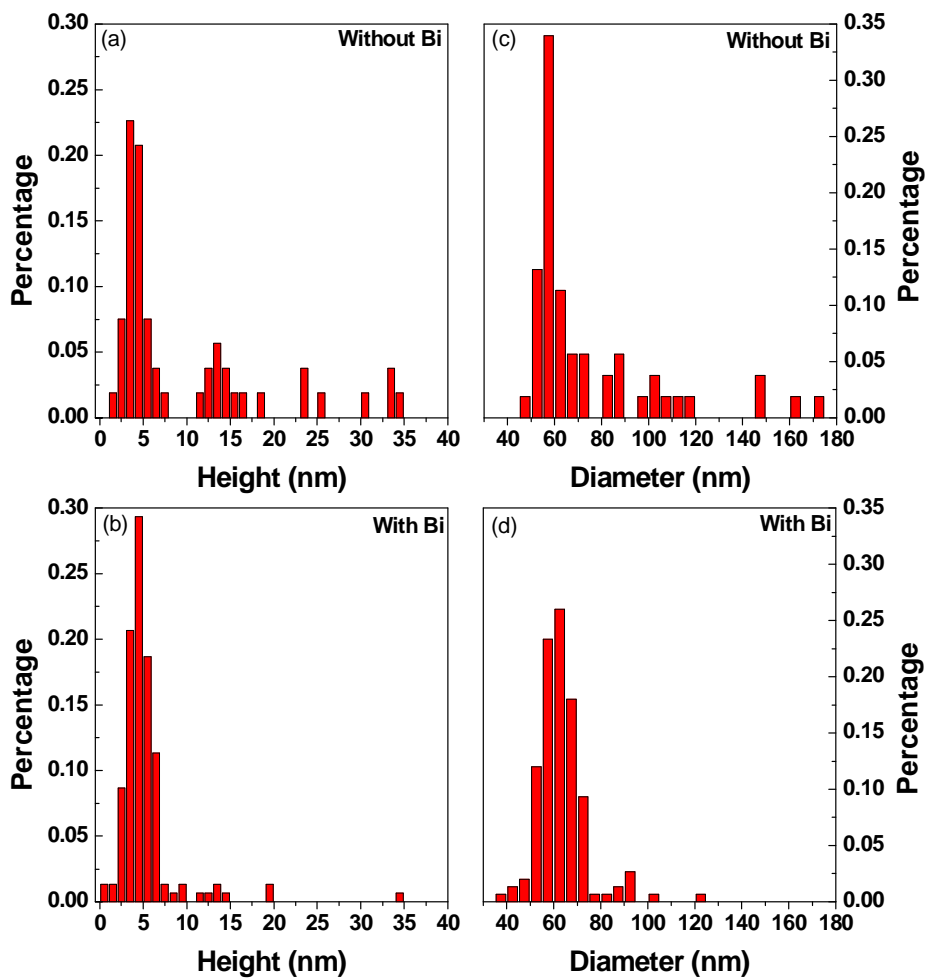

Figure 3. The histograms of the InAs QD height and diameter distribution grown at $500{ }^{\circ} \mathrm{C}$ with and without $\mathrm{Bi}$ exposure.

To analyze the effects of the Bi surfactant on QD uniformity, the typical height and diameter histograms of the InAs QDs grown at $500{ }^{\circ} \mathrm{C}$ with and without $\mathrm{Bi}$ exposure were extracted from the AFM images, as shown in figure 3. From the histograms of height distribution, as shown in figures 3(a) and 3(b), an extremely large proportion of QDs with the height beyond $12 \mathrm{~nm}$ was suppressed markedly after the $\mathrm{Bi}$ exposure. The dot height in the presence of Bi showed less size fluctuation than that without $\mathrm{Bi}$. The average heights were deduced to be 6.3 and $10.5 \mathrm{~nm}$ for InAs QDs with and without Bi exposure, respectively. Similarly, QDs grown without Bi 157 exhibited a wider distribution in the diameter, as shown in figure 3(c). While the fluctuation in the diameter was diminished for the InAs QDs in the presence of Bi, 
and the average QD diameters were deduced to be 64.0 and $74.2 \mathrm{~nm}$ for InAs QDs

160 with and without $\mathrm{Bi}$ exposure, respectively. This means that the $\mathrm{Bi}$ surfactant 161 decreased the typical QD size through suppressing the free surface diffusion of In 162 adatoms during InAs QD growth. This also coincides with the QDs grown with Sb as 163 a surfactant [18, 19].

164

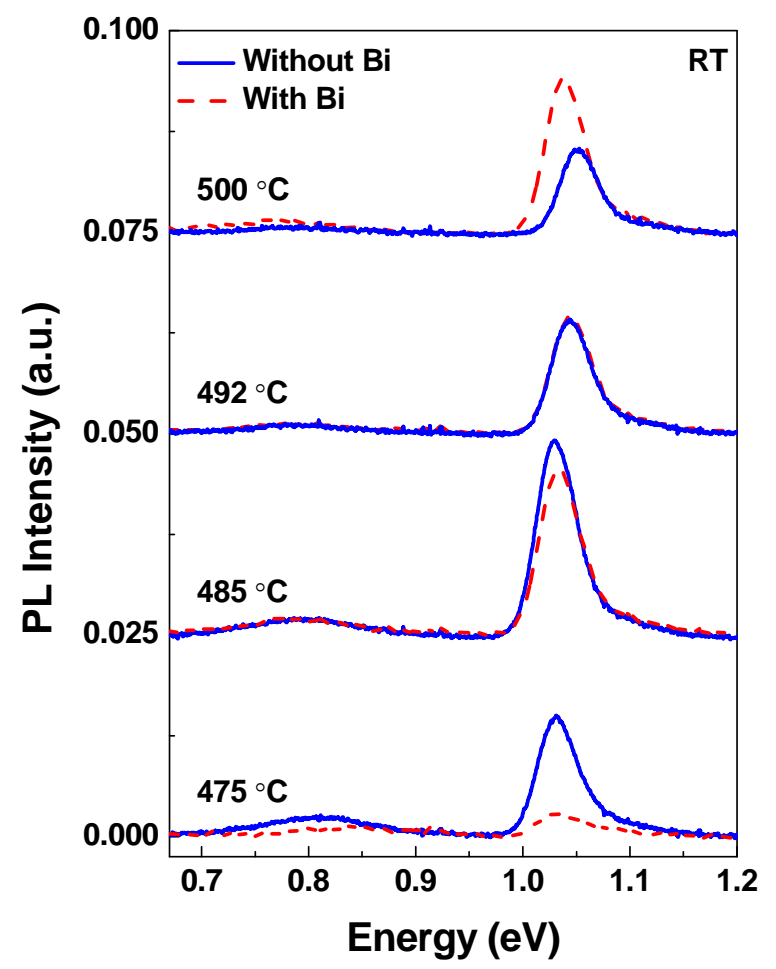

Figure 4. PL spectra of InAs QDs grown at different temperatures with and without Bi exposure.

Figure 4 shows the room temperature (RT) PL spectra for the InAs QDs with and without Bi exposure deposited at different temperatures. The output voltage of the DPSS laser was set to $2.5 \mathrm{~V}$ with an excitation power of about $340 \mathrm{~mW}$. As shown in figure 4, the ground state emission occurred at about $1.03 \mathrm{eV}$ for both sets of QD structures at RT. The broad signal located in the $0.75-0.87 \mathrm{eV}$ range fell off linearly as the temperature increased, corresponding well with the features of the lateral 
associated InAs QDs [20, 21]. It is noticeable that the peak position of the ground state emission for the InAs QDs with $\mathrm{Bi}$ exposure shifted slightly to the red, especially for the InAs QDs and the GaAs capping layer grown at higher temperatures $\left(500{ }^{\circ} \mathrm{C}\right)$ with respect to those without $\mathrm{Bi}$ exposure as shown in figure 3. This phenomenon can be explained by the fact that QDs will be partly dissolved with a lower height during the capping process [22, 23], and as a result their height is lower, but the presence of $\mathrm{Bi}$ atoms can definitely influence this process by suppression of QD dissolution and then increase the height of resulting QDs, which is similar to $\mathrm{Sb}$ atoms $[14,15]$. As a result, this shifts the wavelength of InAs QDs grown with Bi to lower energies. It is worth noting that the bigger hillocks may not show any PL since they are relaxed and contain-annihilation centers_dislocations. The PL spectrum originated only from the small QDs with the height not bigger than $10 \mathrm{~nm}$ - the first maximum in the histogram for both samples shown in figures 3 (a) and (b). This redshift of PL agrees well with the first maximum shifted to bigger QD size for sample grown with $\mathrm{Bi}$ at higher temperatures, as shown in figure 5.

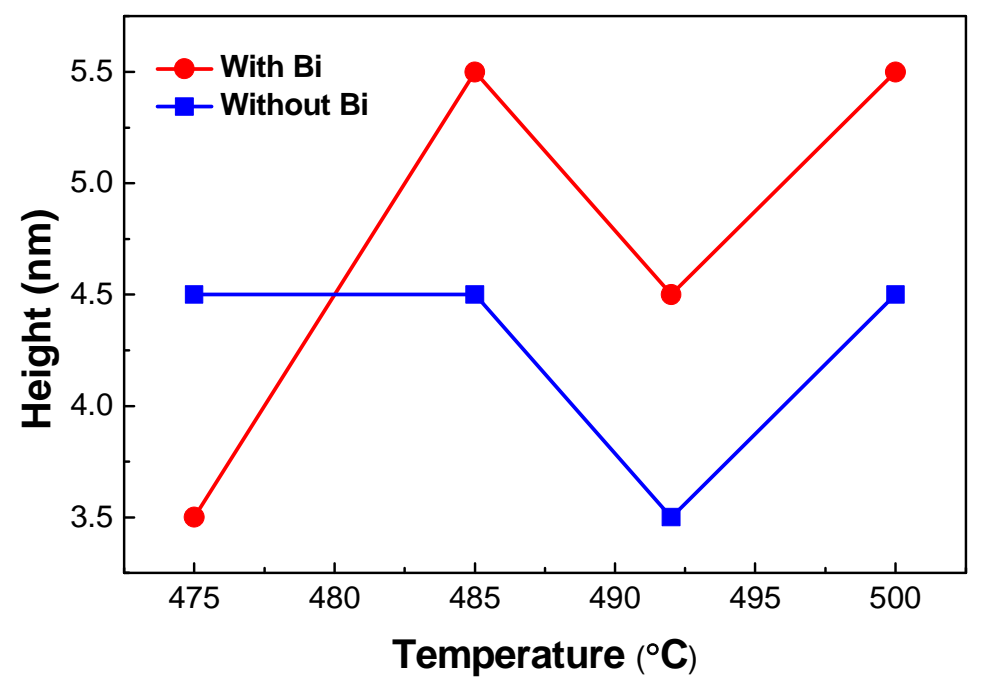

Figure 5. The peak height value for smaller QDs and its dependence on the 
temperature.

Additionally, the PL intensity of the ground state transition for InAs/GaAs QDs with $\mathrm{Bi}$ exposure was weaker at a deposition temperature of $475{ }^{\circ} \mathrm{C}$ but comparable at temperatures of $485-500{ }^{\circ} \mathrm{C}$ with respect to those without $\mathrm{Bi}$ exposure. Since Bi induced layer-by-layer growth in the InAs/GaAs strained systems by reducing the interface energy, and thus the three-dimensional (3D) islands in the Stranski-Krastanov (S-K) growth mode was suppressed. This would lead to postponement of the wetting layer formation before the growth mode changing from $2 \mathrm{D}$ to $3 \mathrm{D}$ during the $\mathrm{S}-\mathrm{K}$ growth process due to the decrease in the strain energy [9, 24]. Then the critical thickness of the wetting layer would be extended, which delayed the formation of InAs QDs. If this wetting layer was modified properly, it would improve the optical property of InAs QDs, but a much thicker one, in turn, could damage it by serving as a channel for thermally activated carriers [25]. The influence could change with the growth temperature due to the variation of the critical thickness of the wetting layer. The inferior PL of InAs QDs grown with Bi exposure at $475{ }^{\circ} \mathrm{C}$ could be caused by the insufficiently developed QDs at this low temperature with the presence of $\mathrm{Bi}$ atoms. 

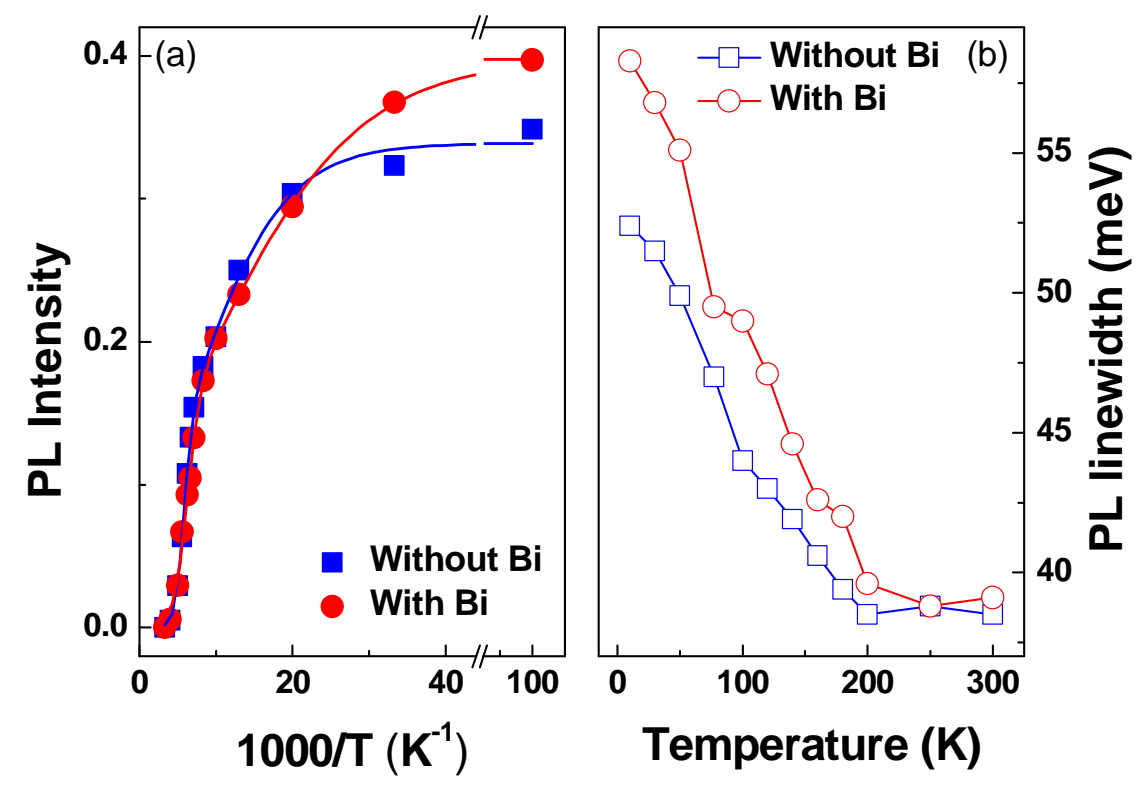

209

Figure 5 6. (a) Integrated PL intensities for InAs QDs grown at $492{ }^{\circ} \mathrm{C}$ with and without $\mathrm{Bi}$ exposure as a function of reciprocal temperature from $10 \mathrm{~K}$ to $300 \mathrm{~K}$. The lines are the least squares fit of the data. (b) PL linewidths for InAs QDs with and without Bi exposure versus temperatures. The lines are drawn as a guide.

To have a deeper understanding of the effect of Bi surfactants on the optical properties of QDs, the change of carrier confinement in both sets of QDs should be investigated further by measuring temperature-dependent PL quenching processes. Therefore, the InAs QDs grown at $492{ }^{\circ} \mathrm{C}$ with similar RT PL spectra under both Bi-mediated and unmediated conditions to eliminate the impact coming from different dot densities were measured. The integrated PL intensities, excited under low-pump conditions, were plotted in figure $5 \underline{6}(\mathrm{a})$ as a function of reciprocal temperature. The fits are derived by applying equation [26], $I(T)=I(0) /\left[1+C_{l} \exp \left(-E_{l} / k_{B} T\right)+\right.$ $\left.C_{2} \exp \left(-E_{2} / k_{B} T\right)\right]$ (Eq. (1)), where $C_{1}$ and $C_{2}$ represent the strengths of the both quenching processes, $I(T)$ and $I(0)$ are the PL integrated intensity at temperature $T$ and $0 \mathrm{~K}, k_{B}$ is Boltzmann' constant and $E_{1}, E_{2}$ are the thermal activation energies 
corresponding to the highest required energies for carriers to escape from the active region in low- and high- temperature regions respectively. Experimental data were well fitted as shown in figure $5 \underline{6}(a)$, and the fitting parameters calculated from the Arrhenius plots were listed in table 1 .

It can be observed that calculated activation energies were 14.6 and $9.0 \mathrm{meV}$ in low-temperature regions, 156.1 and $105.0 \mathrm{meV}$ in high-temperature regions for the InAs QDs without and with $\mathrm{Bi}$ exposure respectively. The reduction in activation energies of $E_{1}$ and $E_{2}$ suggested weaker carrier confinement for InAs QDs with Bi exposure. This reduced confinement potential can be explained by the fact that the size of QDs at lower temperatures with Bi as surfactant is smaller, which decreases the confinement barrier for carriers in QDs [11].

Lastly, the PL linewidths for InAs QDs grown at $492{ }^{\circ} \mathrm{C}$ with and without $\mathrm{Bi}$ exposure as a function of temperature from 10 to $300 \mathrm{~K}$ were plotted in figure $5 \underline{6}(\mathrm{~b})$. The linewidths of both spectra substantially increased in low-temperature regions due to the appearance of emission from small QDs [27]. While at high temperatures, the QDs with high localization energies will be preferentially occupied by carriers and thus dominated the spectra, resulting in narrow linewidths. It is noted that the linewidths were broadened lightly for QDs with Bi exposure over the temperature range of 10-300 K, which also indicates that a weak carrier confinement occurred in the QDs grown with the presence of Bi.

Table 1. Fitting parameters for the measured temperature-dependent integrated PL intensities of InAs QDs with and without Bi. 
Table 1.

\begin{tabular}{lccccc}
\hline \hline Sample & $I(0)$ & $E_{1}$ & $C_{1}$ & $E_{2}$ & \multicolumn{2}{l}{$C_{2}$} \\
\hline Without Bi & 0.34 & 14.6 & 3.4 & 156.1 & 73943 \\
With Bi & 0.40 & 9.0 & 2.7 & 105.0 & 3951.9 \\
\hline \hline
\end{tabular}

249

\section{Conclusions}

The influence of Bi exposure on the self-assembled InAs/GaAs QDs has been investigated by comparison of dot density and dimension at varying substrate temperatures. It is shown that the dot density decreases but large, defective InAs islands accumulate as the growth temperature increases under the Bi-unmediated condition. By contrast, for the Bi-mediated growth, the dot areal density decreases at low temperatures but increases at high temperatures, which reveals essentially the suppression effect of $\mathrm{Bi}$ on the surface migration and desorption of In adatoms. Furthermore, the QD dimensions become more uniform and homogeneous at higher temperatures for $\mathrm{Bi}$ atoms suppressing the formation of larger dislocated islands. If the negative effect of $\mathrm{Bi}$ can be avoided, Bi-mediated QDs could show great application potential for photonic and optoelectronic devices in the future.

\section{Acknowledgments}

The authors wish to acknowledge the support of the National Key Research and Development Program of China under Grant No. 2016YFB0402400, the National Natural Science Foundation of China under grant Nos. 61675225, 61605232 and 61775228. 


\section{References}

269 [1] Sakamoto K, Kyoya K, Miki K, Matsuhata H and Sakamoto T 1993 Which

Surfactant Shall We Choose for the Heteroepitaxy of Ge/Si(001)? -Bi as a Surfactant 271 with Small Self-Incorporation- Jpn. J. Appl. Phys. 32 L204-L206.

272

[2] Okamoto H, Tawara T, Gotoh H, Kamada H and Sogawa T 2010 Growth and

Characterization of Telecommunication-Wavelength Quantum Dots Using Bi as a

Surfactant Jpn. J. Appl. Phys. 49 06GJ01.

[3] Gu Y, Zhang Y G, Chen X Y, Xi S P, Du B and Ma Y J 2015 Effect of bismuth Phys. Lett. 107212104.

[4] Paul N, Asaoka H, Myslivecek J and Voigtlander B 2004 Growth mechanisms in $\mathrm{Ge} / \mathrm{Si}(111)$ heteroepitaxy with and without $\mathrm{Bi}$ as a surfactant Phys. Rev. B 69 193402.

[5] Polit A, Kac M, Krupinski M, Samul B, Zabila Y and Marszalek M 2007 The Behaviour of Surfactants during the Growth of $\mathrm{Co} / \mathrm{Cu}$ Multilayers Acta Phys. Pol. A $1121281-1287$.

[6] Yang Y, Jo B, Kim J, Lee C R, Kim J S, Oh D K, Kim J S and Leem J Y 2009 Optical stability of shape-engineered InAs/InAlGaAs quantum dots J. Appl. Phys. 105 053510.

[7] Saito H, Nishi K, Kamei A and Sugou S 2000 Low chirp observed in directly modulated quantum dot lasers IEEE Photon. Technol. Lett. 12 1298-1300.

[8] Chen S M et al 2016 Electrically pumped continuous-wave III-V quantum dot 
290

291

292

293

294

295

296

297

298

299

300

301

302

303

304

305

306

307

308

309

310

311

lasers on silicon Nat. Photonics 10 307-311.

[9] Dasika V D, Krivoy E M, Nair H P, Maddox S J, Park K W, Jung D, Lee M L, Yu

E T and Bank S R 2014 Increased InAs quantum dot size and density using bismuth as a surfactant Appl. Phys. Lett. 105253104.

[10] Zvonkov B N, Karpovich I A, Baidus N V, Filatov D O, Morozov S V and Gushina Y Y 2000 Surfactant effect of bismuth in the MOVPE growth of the InAs quantum dots on GaAs Nanotechnology 11 221-226.

[11] Fan D S, Zeng Z Q, Dorogan V G, Hirono Y, Li C, Mazur Y I, Yu S Q, Johnson S R, Wang Z M, Salamo G J 2013 Bismuth surfactant mediated growth of InAs quantum dots by molecular beam epitaxy J Mater Sci: Mater Electron 24 1635-1639.

[12] Massies J and Grandjean N 1993 Surfactant effect on the surface diffusion length in epitaxial growth Phys. Rev. B $\mathbf{4 8} 8502$.

[13] Feng G, Oe K, Yoshimoto M 2007 Temperature dependence of Bi behavior in MBE growth of InGaAs/InP J. Cryst. Growth 301-302 121-124.

[14] Matsuura T, Miyamoto T, and Koyama F 2006 Topological characteristics of InAs quantum dot with GaInAs cover using Sb surfactant Appl. Phys. Lett. 88 183109.

[15] Guimard D, Nishioka M, Tsukamoto S, Arakawa Y 2007 Effect of antimony on the density of InAs/Sb:GaAs(100) quantum dots grown by metalorganic chemical-vapor deposition J. Cryst. Growth 298 548-552.

[16] Chu L, Arzberger M, Böhm G and Abstreiter G 1999 Influence of growth conditions on the photoluminescence of self-assembled InAs/GaAs quantum dots $J$ 
Appl. Phys. 852355.

[17] Ajayan P M, Carlson J, Bayburt T and Nalwa H S (editor) 2002 Nanostructured materials and nanotechnology (Academic Press).

[18] Kakuda N, Yoshida T and Yamaguchi K 2008 Sb-mediated growth of high-density InAs quantum dots and GaAsSb embedding growth by MBE Appl. Surf. Sci. 254 8050-8053.

[19] Guimard D, Nishioka M, Tsukamoto S and Arakawa Y 2007 Effect of antimony on the density of InAs/Sb:GaAs(100) quantum dots grown by metalorganic chemical-vapor deposition J. Cryst. Growth 298 548-552.

[20] Zhukov A E, Volovik B V, Mikhrin S S, Maleev N A, Tsatsul'nikov A F, Nikitina

E V, Kayander I N, Ustinov V M and Ledentsov N N 2001 1.55-1.6 $\mu \mathrm{m}$ electroluminescence of GaAs based diode structures with quantum dots Tech. Phys. Lett. 27 734-736.

[21] Tonkikh A A, Cirlin G E, Talalaev V G, Novikov B V, Egorov V A, Polyakov N K, Samsonenko Yu B, Ustinov V M, Zakharov N D, Werner P 2003 Room-temperature $1.5-1.6 \mu \mathrm{m}$ photoluminescence from InGaAs/GaAs heterostructures grown at low substrate temperature Semiconductors 37 1406-1410.

[22] Hospodková A, Vyskočil J, Pangrác J, Oswald J, Hulicius E, Kuldová K 2010 Surface processes during growth of InAs/GaAs quantum dot structures monitored by reflectance anisotropy spectroscopy Surf. Sci. 604 318-321.

[23] Utrilla A D et al 2018 Size and shape tunability of self-assembled InAs/GaAs nanostructures through the capping rate Appl. Surf. Sci. 444 260-266. 
334 [24] Matsuura T, Miyamoto T, Kageyama T, Ohta M, Matsui Y, Furuhata T and 335 Koyama F 2004 Surfactant Effect of Sb on GaInAs Quantum Dots Grown by 336 Molecular Beam Epitaxy Jpn. J. Appl. Phys. 43 L605-L607.

337 [25] Kudrawiec R and Misiewicz J 2009 Photoreflectance and contactless 338 electroreflectance measurements of semiconductor structures by using bright and dark 339 configurations 2009 Rev. Sci. Instrum. 80096103.

340 [26] Gu Y, Zhang Y G, Li A Z, Wang K, Li C and Li Y Y 2009 Structural and 341 Photoluminescence Properties for Highly Strain-Compensated InGaAs/InAlAs 342 Superlattice Chin. Phys. Lett. 26077808.

343 [27] Bouzaïene L, Saidi F, Sfaxi L and Maaref H 2010 Temperature dependence of the 344 optical properties of InAs quantum dots with bimodal size evolution grown on GaAs 345 (115)A substrate Physica B 405 744-747. 